\title{
EL DESARROLLO PSICOLÓGICO HUMANO COMO PROCESO DE CONTINUIDAD Y RUPTURA: LA "SITUACIÓN SOCIAL DEL DESARROLLO"
}

Laura Domínguez García*

\begin{abstract}
Resumen
Presentamos una caracterización de la Edad Escolar, la Adolescencia y la Juventud en base a la "situación social del desarrollo" como categoría básica del Enfoque Histórico Cultural, la cual nos permite mostrar como el desarrollo psicológico humano es un proceso de continuidad y ruptura, un proceso revolucionario que ocurre a saltos, un proceso donde se producen cambios cualitativos y que transcurre en espiral hacia niveles superiores de conquista de la autodeterminación por parte del sujeto, en función de transformaciones que se producen en los sistemas de actividades y comunicación en los que se desarrolla su vida y en las principales formaciones psicológicas que se consolidan hacia el final de cada edad conservando la historia de su desarrollo.
\end{abstract}

Palabras claves: Situación social del desarrollo. Sistemas de actividades y comunicación. Formaciones psicológicas.

\begin{abstract}
In this paper, we stand a characterization of primary school Age, Adolescence and Youth based on the "social situation of development" as a basic category of Historical Cultural approach, which allows us to show how the human psychological development is a process of continuity and break, a revolutionary process discontinuously, a process in which qualitative changes occur and set in spiral to higher levels of acquisition of self-determination by the subject, based on transformations happening in systems of communication and activities and main psychological formations consolidated at the end of each age preserving the history of its development.
\end{abstract}

Key words: Social situation of development. Systems of communication and activities. Psychological formations.

* Doutora em Ciências Psicologicas pela Universidade de Havana. Professora na Faculdade de Psicologia da Universidade de La Habana, Cuba.E-mail: ldominguez@psico.uh.cu 


\section{Introducción}

El principal propósito de este trabajo es presentarles - en apretada síntesis - cómo apoyándonos en la categoría de "situación social del desarrollo" se muestran las principales tendencias del desarrollo psicológico humano en las diferentes etapas que abarca este proceso, lo que avala el valor explicativo - teórico y metodológico- de dicha categoría para la interpretación de las regularidades del desarrollo en cualquier edad psicológica.

A partir del análisis de la "situación social del desarrollo" podemos mostrar que el desarrollo psicológico humano es un proceso de continuidad y ruptura, un proceso revolucionario que ocurre a saltos, aun cuando tiene momentos de retroceso; un proceso que analizado desde una perspectiva epistemológica materialista y dialéctica, va en una dirección progresiva, no tratándose de una simple sumatoria o acumulación de cambios cuantitativos, en tanto sus fuerzas motrices son las contradicciones; un proceso donde se producen cambios cualitativos que transcurre en espiral hacia niveles superiores de conquista de la autodeterminación por parte del sujeto, conservando la historia de su desarrollo.

En mi interpretación, la "situación social del desarrollo" como categoría básica del enfoque histórico cultural (VYGOTSKI, 1987; 1984; $1982 ; 1935)$ apunta a las transformaciones que se producen en los sistemas de actividades y comunicación en los que se desarrolla la vida del sujeto, así como a las principales adquisiciones del desarrollo psicológico y de las formaciones de la personalidad que se consolidan hacia el final de cada período; de ahí su significación teórica y metodológica para la Psicología del Desarrollo, como disciplina científica empeñada en descubrir las regularidades de este proceso (DOMÍNGUEZ, 2007).

En función de las consideraciones anteriores presentaremos a continuación una caracterización de la Edad Escolar, la Adolescencia y la Juventud, por ser aquellas que resultan más cercanas a mi práctica profesional y en las cuales se producen cambios significativos en el proceso de formación de la personalidad.

\section{Sistema de actividades}

La edad escolar se inicia con el ingreso en la escuela, importante momento en el proceso de desarrollo de la personalidad, el cual trae aparejado 
un cambio trascendental de la manera en que transcurría su vida del niño en el período anterior; es decir, en la edad preescolar. En cuanto al sistema de actividades surge por primera vez una actividad formal o institucionalizada. Nos referimos en este caso a la actividad de estudio. La actividad de estudio, núcleo de la actividad escolar, es una actividad seria, socialmente importante y obligatoria. Su principal objetivo es promover la asimilación por parte del niño de un sistema de conocimientos, hábitos y habilidades, así como la formación de cualidades psicológico-morales. La escuela se convierte entonces en el primer peldaño del proceso de preparación del niño para lograr determinada "competencia" en su futura vida adulta.

En la adolescencia vemos que el adolescente continúa asistiendo a las instituciones de la enseñanza media con carácter obligatorio. Sin embargo, y a diferencia lo que acontece en la edad escolar, en la adolescencia la actividad de estudio cambia tanto por su contenido como por su forma e impone al adolescente la necesidad de utilizar nuevos métodos de asimilación de los conocimientos, proceso que se aun torna más complejo en la edad juvenil.

En la juventud la actividad de estudio adquiere un carácter científico-profesional sobre todo para aquellos jóvenes que continúan desarrollando sus estudios en la Educación Superior. En este caso, deberán asimilar contenidos pertenecientes a diversas disciplinas, los cuales presentan un elevado nivel de abstracción y generalización. También el joven puede decidir dedicarse por entero a determinada actividad laboral o combinar el estudio con el trabajo, y no puede obviarse la posibilidad de que existen jóvenes desvinculados de ambas actividades, por lo que en la juventud es más diverso el espectro en el análisis de todos los componentes de la "situación social del desarrollo".

En lo relativo a la actividad informal o no institucionalizada, tenemos que en la edad escolar el niño comienza a participar en actividades extraescolares de diversos tipos (científicas, deportivas, culturales y recreativas), algunas de ellas, como en el caso de nuestro país, como resultado de su pertenencia a la organización de pioneros, lo que contribuye al desarrollo de intereses variados. La actividad de juego se complejiza y amplía, ya que continúa desarrollándose el juego de roles propio de la edad preescolar y, además, aparece un nuevo tipo de juego: el juego de reglas.

En la adolescencia la elección de las actividades que se realizan en el tiempo libre está condicionada en gran medida por los intereses del grupo de coetáneos. Puede suceder que alguna de estas actividades sea altamente 
motivante para el adolescente y llegue a relegar las actividades vinculadas a la escuela a un segundo plano. También pueden encontrase o no vinculadas con la profesión a la que piensan dedicarse en el futuro.

En la juventud la vida cultural y recreativa se encuentra relacionada fundamentalmente a espacios externos al centro de estudios o laboral. Según I. S. Kon (1990), la lectura, el cine y la televisión se encuentran entre las actividades preferidas en este grupo evolutivo y, en ocasiones, su gran dispersión de intereses les ocasiona dificultades en la organización y distribución de su tiempo. En investigaciones realizadas bajo nuestra dirección se constatan estas limitaciones en lo relativo a la planificación y empleo del tiempo (ÁGUILA, 2005; HERNÁNDEZ, 2006).

\section{Sistema de comunicación}

En su trabajo titulado "La crisis de los siete años" Vygotski (COLECTIVO DE AUTORES, 2003, p. 60) hace referencia a los cambios que se producen en el organismo del niño alrededor de esta edad, ya que es “... la edad del cambio de dientes, del estirón”. Estos cambios biológicos, unidos a las nuevas condiciones que enfrenta el niño en sus sistemas de actividad y comunicación, poseen una importante repercusión psicológica. Al respecto escribe: "El rasgo más importante de la crisis de los siete años es, a nuestro juicio, la diferenciación incipiente de la faceta interior y exterior de la personalidad del niño", que provoca una "intensa pugna de vivencias." (VYGOTSKI en COLECTIVO DE AUTORES, 2003, p. 61). El principal resultado de este proceso en la edad escolar es pérdida de la espontaneidad e ingenuidad típicas del pre-escolar.

La pérdida de la espontaneidad significa que incorporamos a nuestra conducta el factor intelectual que se inserta en la vivencia y en el acto directo, lo que viene a ser el polo opuesto de la acción ingenua y directa propia del niño (COLECTIVO DE AUTORES, 2003, p. 61).

Esta nueva potencialidad del desarrollo infantil es calificada por Vygotski como "vivencia atribuida de sentido", en la medida en que por primera vez el niño tiene conciencia del significado de sus vivencias, estas se generalizan y aparece la "lógica de los sentimientos". 
El sistema de interrelaciones con quienes le rodean coloca al escolar en una nueva "posición social", condicionada no de manera lineal por su "posición objetiva", sino por un conjunto de exigencias que se derivan de ella. En este sistema de comunicación destacaremos, en primer término, las relaciones con los adultos. En la escuela aparece una nueva figura de gran importancia en el sistema de interrelaciones del niño, cuya valoración acerca de la conducta del escolar tendrá un gran impacto en la esfera emocional del mismo, sobre todo en los primeros grados. Nos referimos en este caso al maestro, quien desde el ingreso del niño en la escuela y aproximadamente hasta el 4to Grado se convierte en una autoridad sagrada para los escolares (BOZHOVICH, 1976). Sus criterios influirán de forma decisiva en el desarrollo de la autovaloración del escolar, y de la aceptación o rechazo que el maestro le demuestre al niño, dependerá, en buena medida, el bienestar emocional de este.

Por su parte los padres también exigen al escolar el cumplimiento de sus responsabilidades. La actitud que asuman ante la ejecutoria de su hijo en la escuela contribuirá a estimular o disminuir los intereses del escolar por la actividad de estudio. Se ha constatado que muchos problemas de aprendizaje y determinados problemas de conducta frecuentes entre los escolares se asocian a situaciones familiares inadecuadas, donde existen conflictos, ausencia de las figuras paternas, carencia afectiva, etc.

Al analizar el sistema de comunicación en la adolescencia observamos que resulta prácticamente inevitable que se produzcan conflictos entre adolescentes y adultos. A esto se suma el hecho de que a partir del 4to grado el ocupar el lugar al que aspira el escolar dentro de su grupo de coetáneos, comienza a convertirse en el motivo fundamental de la conducta (BOZHOVICH, 1976). Por esta razón, los adultos dejan de ser autoridades sagradas en comparación con la etapa precedente.

Los adolescentes desarrollan una mayor criticidad en la valoración de los maestros y de sus padres. Esta valoración es poco reflexiva y también tiende a ser rígida, dado el carácter dicotómico del pensamiento en este período, así como por la insuficiente consolidación de algunas formaciones motivacionales de la personalidad. En el caso de los maestros su aceptación por parte de los adolescentes va a depender en mayor medida de su estilo de comunicación que de su preparación técnica, entendida como excelencia académica en la materia o materias que imparte. En cuanto a las relaciones con los padres podemos señalar que al inicio de la etapa el adolescente 
continúa muy centrado en su seno familiar, pero al producirse los cambios biológicos - también denominados transformaciones puberales-, y en especial el proceso de maduración sexual, tanto adolescentes como padres valoran el mayor nivel de madurez logrado por los primeros y sus potencialidades para la independencia (YEMI, 2006; HUMARAN, 2009).

El conflicto adulto-adolescente (PETROVSKY, 1980) tiene como principal causa la divergencia de opiniones de adultos y adolescentes en cuanto a los derechos y deberes de estos últimos. Esta divergencia se encuentra condicionada por factores objetivos y subjetivos: el adolescente ocupa una posición social intermedia, continúa dependiendo económicamente de los padres, aún asiste a una institución escolar con vistas a lograr la preparación necesaria para su futuro $y$, en ocasiones, muestra conductas infantiles o rasgos de inmadurez. Para atenuar este fenómeno o solucionarlo se deben producir cambios en el estilo de comunicación del adulto hacia el adolescente. El adulto debe combinar la dirección firme y consecuente de sus exigencias al comportamiento del adolescente, con la flexibilidad necesaria y racionalidad de estas.

Una vez analizado en el conflicto adulto-adolescente pasamos a caracterizar la llamada crisis de la adolescencia. Para los psicólogos de orientación marxista la crisis de la adolescencia es una crisis de carácter psicológico, en tanto reflejo cognitivo y vivencial de la falta de correspondencia que se produce entre las crecientes potencialidades físicas y psicológicas del adolescente y las posibilidades reales con que cuenta para su realización, en el contexto de los sistemas de actividades y comunicación donde transcurre su vida. La crisis es valorada como fuerza motriz del desarrollo, por lo que los psicólogos marxistas enfatizan en su aporte o influencia favorable sobre este proceso.

En la jventud las relaciones con los adultos se basan igualmente en la valoración crítica de sus cualidades psicológicas y morales que realiza el joven de sus padres y maestros, pero esta valoración posee un mayor nivel de argumentación que en la adolescencia y es mucho más flexible. En cuanto a los maestros, los jóvenes tienen en cuenta su competencia profesional, además de sus cualidades personales y su estilo de comunicación, prefiriendo el que se sustenta en el diálogo y el respeto mutuo. En lo que respecta a la relación con los padres, el joven logra una mayor independencia emocional de la familia en comparación con el adolescente. Los padres 
constituyen modelos de conducta, pero de manera más mediatizada que en etapas anteriores. O. Kraftchenco (Citada por Domínguez G., L., 2003a) se observó que los padres constituyen modelos subjetivos para la estabilidad del comportamiento moral y específicamente de la conducta honesta, en aquellos jóvenes que poseen un mayor nivel de desarrollo moral.

Continuando nuestro análisis podemos señalar que el sistema de comunicación incluye, de forma especialmente relevante, las relaciones con los coetáneos o iguales. En la edad escolar la posición que el niño ocupa en el grupo de coetáneos o iguales y su sistema de interrelaciones tienen una importante influencia en el desarrollo de su personalidad, sobre todo a partir del 4to grado. En este sentido, L. I. Bozhovich (1976) considera que el encontrar en el grupo el lugar al que se aspira se convierte en motivo fundamental de la conducta a finales de la Edad Escolar. Esta motivación por alcanzar el lugar deseado entre los iguales, como veremos posteriormente, pasa a un primer plano en la adolescencia.

En la Adolescencia los sujetos pasan más tiempo con sus compañeros que en el seno familiar, por lo que sus valores y normas de conducta van a estar determinados, en buena medida, por el carácter de estas interrelaciones, tanto en el grupo formal como en el espontáneo o informal. La necesidad de ocupar el lugar deseado o lugar al que aspira en su grupo de coetáneos o iguales, desempeña un importante papel en el desarrollo armonioso de la personalidad en esta etapa, ya que el equilibrio y bienestar emocional del adolescente dependerá de que logre este propósito y de la aceptación que alcance dentro del grupo. L. I. Bozhovich (1976) explica diferentes fenómenos del comportamiento de los adolescentes como son el conformismo y el negativismo y la llamada "persecución de la nota". Muchos adolescentes se empeñan en obtener altas calificaciones en la escuela, aún cuando éstas no sean resultado de su preparación académica ni de sus conocimientos, como medio de obtener prestigio entre sus compañeros de clase. Esta necesidad conduce en ocasiones al fraude académico. La ausencia del reconocimiento deseado por parte de sus iguales puede llevar al adolescente a convertirse en el peor alumno de su aula y por este camino a conductas antisociales que pueden desembocar en la comisión de actos delictivos. Investigaciones realizadas por M. A. Alemanski y G. G. Bochkarieva (KON, 1990) así lo demuestran.

El desarrollo de la capacidad de autodeterminación en la juventud y la necesidad de independencia mejor estructurada y fundamentada, a diferencia de lo que ocurre en la adolescencia, permiten al joven mostrarse como un 
ser relativamente independiente de las opiniones de su grupo de coetáneos o iguales, siendo capaz de enfrentarse abiertamente a determinados criterios, si considera inadecuadas ciertas posiciones, normas o exigencias grupales. No obstante, de manera semejante a lo descrito para la etapa de la adolescencia, estos grupos se convierten en importantes espacios de reflexión, de expresión y conformación de sus puntos de vista y, además, contribuyen a la organización y empleo de su tiempo libre.

La amistad constituye otra importante esfera en el sistema de comunicación. En la edad escolar las relaciones de amistad son poco profundas e inestables y la selección del amigo es poco intencional, sobre todo al inicio de la etapa, respondiendo a criterios externos y un tanto casuales, como pueden ser el que dos niños sean vecinos o se sienten cerca en el aula por disposición de la maestra.

Durante la adolescencia esta relación de amistad se basa en la confianza total, la ayuda, el respeto mutuo y se produce una tendencia a idealizar al amigo por lo que la amistad puede romperse con relativa facilidad. Este hecho está en consonancia con el desarrollo intelectual, porque el pensamiento, como posteriormente veremos, tiende a ser dicotómico y poco flexible. Para D. B. Elkonin (1987), de acuerdo al sistema de periodización del desarrollo psíquico que propone, la actividad rectora en este período es la relación íntimo-personal adolescente-adolescente. Esta relación comunicativa, se distingue por encontrarse basada en un código de camaradería que regula las relaciones entre los adolescentes, bajo las premisas de la ayuda mutua, la confianza total y el respeto. Este código de comunicación se expresa con mayor fuerza y claridad en las relaciones de amistad.

En la juventud se produce una búsqueda intensa de la amistad, la cual es concebida como una relación afectiva, altamente individualizada, estable y profunda, se rigen por importantes valores morales, como son la honestidad y la lealtad; y a diferencia de la adolescencia, la amistad presenta un carácter más selectivo, diferenciándose los compañeros de los amigos.

Pasando a caracterizar las relaciones de pareja como componente del sistema de comunicación tenemos que en la edad escolar no existen relaciones de pareja, en el sentido tradicional del término, aunque los escolares pueden "enamorarse" platónicamente de un adulto o coetáneo.

En el transcurso de la adolescencia la aparición de la relación de pareja comienza a relegar las relaciones de amistad a un segundo plano. Las 
relaciones de pareja en esta etapa tienden a ser inestables, ya que poseen un carácter experimental y contribuyen al desarrollo de la autovaloración o identidad personal y, en particular, de la identidad sexual y de género del adolescente. Esto resulta peligroso, pues los adolescentes no cuentan -en muchos casos- con una adecuada educación sexual, por lo que se constituyen en grupo de riesgo por excelencia para contraer enfermedades de trasmisión sexual y también pueden llegar a una maternidad o paternidad precoz para la que no están preparados ni objetiva ni subjetivamente.

En la juventud la búsqueda de una mayor estabilidad en las relaciones de pareja, constituye una tendencia, aunque el logro de dicha estabilidad y la forma en que cada miembro de la pareja enfoque y proyecte la relación, dependerá de la educación recibida principalmente en la familia respecto a esta esfera. También aflora como tendencia el proyecto de creación de la familia propia, la preparación para el matrimonio y la llegada del primer hijo.

\section{Nuevas formaciones psicológicas}

\subsection{Desarrollo intelectual}

En la edad escolar podemos destacar como importantes adquisiciones del desarrollo intelectual, en estrecho vínculo con la influencia que ejerce en el desarrollo psicológico del niño la actividad de estudio, el surgimiento del pensamiento conceptual empírico, el desarrollo de los intereses cognoscitivos, y el carácter consciente y voluntario que adquieren los procesos cognoscitivos, lo cual posibilita al escolar una mayor estabilidad en el control efectivo de su comportamiento.

Pasando a analizar el desarrollo del lenguaje podemos decir lo siguiente. El lenguaje hablado de carácter situacional, propio de la edad preescolar, aunque no desaparece totalmente, comienza a ser desplazado por un lenguaje hablado más lógico y coherente. Este lenguaje constituye una premisa y su desarrollo es consecuencia de las exigencias que impone al desarrollo psicológico del niño la actividad de estudio, pues la misma demanda la comprensión por el escolar de diferentes contenidos y la capacidad de expresar lo aprendido de forma comprensible para los demás. Asimismo, por primera vez, el niño comienza a utilizar el lenguaje escrito, hecho que favorece la coherencia del lenguaje hablado, al ponerlo ante la necesidad de estructurar 
gramaticalmente, de forma adecuada, sus expresiones verbales. En esta etapa se unen pensamiento y lenguaje, procesos que venían hasta entonces desarrollándose paralelamente, en opinión de L. S. Vygotski (1982). En la Edad Escolar el pensamiento adquiere un nivel conceptual, lo que significa que este proceso opera a través de conceptos, los cuales se definen mediante las palabras. De esta forma, el lenguaje mantiene su función comunicativa y comienza a poseer también una función intelectual.

Las clases dirigidas a propiciar la asimilación de la lengua materna y, en general, todas las actividades académicas, deben contribuir al desarrollo del lenguaje del escolar, de su riqueza y coherencia, mediante el control de las respuestas del niño, los ejercicios de composición, etc. Para llevar a cabo de forma adecuada esta tarea, el maestro debe contar con un vocabulario amplio y con habilidades comunicativas adecuadas, en cuanto a expresión y dicción.

En lo que respecta al desarrollo del pensamiento podemos señalar que surge el pensamiento conceptual empírico que, según Rubinstein (1967) es abstracto por su forma, ya que opera a través de conceptos, a nivel mental, pero empírico por su contenido puesto que la asimilación de todo nuevo conocimiento debe partir de lo directamente perceptible, ya sea del objeto en cuestión o su materialización (representación). A su vez Piaget (1966; 1969) califica este pensamiento como operatorio concreto, en tanto surgen un conjunto de nociones y operaciones, como es la noción de conservación de sustancia alrededor de los 7 años, que son acciones interiorizadas y reversibles, las cuales se forman partiendo de situaciones concretas.

Hasta el presente, y en correspondencia con la forma en que se organiza el proceso de enseñanza (tradicional), el pensamiento en la Edad Escolar sigue una lógica inductiva en su proceso de formación, por lo que al inicio de esta edad puede presentarse determinada confusión entre los conceptos científicos y las representaciones de la vida cotidiana que el niño ha ido conformando en su desarrollo precedente. El pensamiento conceptual empírico presenta limitaciones: en ocasiones el niño aunque es capaz de aplicar el concepto no logra definirlo, tiene dificultades para diferenciar lo esencial de lo secundario, así como para establecer comparaciones y tiende a las soluciones estereotipadas. Algunos autores consideran que las limitaciones antes descritas aparecen como una regularidad "natural" y no necesaria del desarrollo intelectual en esta edad (DAVIDOV, 1981; GALPERIN, 1987) 
y demuestran en el plano experimental que estas características varían al transformarse la enseñanza. Por ejemplo, desaparece la mezcla de conceptos científicos con los de la vida cotidiana, que según L. S. Vygotski (1982), se produce en los inicios de la edad escolar. También en nuestro país algunos investigadores han obtenido resultados semejantes (MARTÍNEZ; FARIÑAS apud COLECTIVO DE AUTORES, 1990).

El desarrollo intelectual en la etapa de la adolescencia se encuentra vinculado en buena medida a la influencia ejercida por el sistema de actividades. En cuanto al desarrollo de los intereses cognoscitivos podemos señalar que aumenta la variedad de intereses, y en el tránsito de la adolescencia a la juventud, el sujeto se va tornando cada vez más selectivo e intencional, respecto a las esferas de la realidad hacia las cuales estos se orientan. En la adolescencia surgen intereses profesionales sobre la base de los intereses cognoscitivos, pero como tendencia, son inestables.

Pasando a la caracterización del desarrollo del pensamiento en la Adolescencia podemos decir que el pensamiento conceptual empírico, propio de la Edad escolar, se transforma en pensamiento conceptual teórico, al decir de S. L. Rubinstein (1967). A este tipo de pensamiento J. Piaget (1966; 1969) lo denomina inteligencia operatoria formal o estadio de las operaciones formales. La principal limitación del pensamiento del adolescente es su carácter dicotómico; es decir, su rigidez, en tanto se basa en muchas ocasiones en generalizaciones extremas que obvian los posibles matices en el análisis de cualquier situación.

En la juventud los intereses cognoscitivos se encuentran un tanto supeditados a los intereses profesionales, aunque son variados y responden también a las necesidades culturales del joven. Los jóvenes se orientan hacia el contenido de las asignaturas en mayor grado que hacia sus aspectos externos y prefieren aquellas que promueven su reflexión y los conducen a realizar generalizaciones, a partir de las cuales, pueden dar explicación a determinados hechos concretos. En este período se consolidan los intereses profesionales y pueden convertirse en intenciones profesionales (GONZÁLEZ, 1983a).

En la Juventud se consolida el pensamiento conceptual teórico propio de la adolescencia. Además, coincidimos con L. I. Bozhovich (1976), quien expresara que en la Juventud, este proceso cognoscitivo por excelencia, adquiere un carácter emocional personal. El joven se interesa por la solución de 
problemas cognoscitivos generales y por todo lo relacionado con los valores morales e ideológicos, dada su necesidad de autodeterminación y su aspiración a elaborar una concepción del mundo propia. Algunos autores, desde una valoración crítica del sistema de estadios propuesto por J. Piaget, señalan el surgimiento de un pensamiento pos formal basado en mayor medida en una interpretación de la realidad desde la perspectiva de la lógica dialéctica y no de la lógica formal piagetiana (CARRETERO; LEÓN, 1999).

\subsection{Desarrollo afectivo motivacional}

\subsubsection{La autovaloración o identidad personal}

Un importante componente de la esfera afectivo motivacional que se desarrolla en estas etapas es la autovaloración, también denominada con el término de identidad personal. Este término hace referencia al concepto que tiene el sujeto sobre su propia persona, el cual elabora sobre la base de sus principales necesidades y motivos. Esta formación posee una función subjetivo-valorativa, relativa a la valoración del sujeto sobre sí mismo y una función reguladora, pues lo impulsa a actuar, en correspondencia con la valoración que tiene de su propia persona (GONZÁLEZ, 1983b). En este sentido, se considera que en la edad escolar la función subjetivo-valorativa presenta un mayor desarrollo que la reguladora (ROLOFF apud COLECTIVO DE AUTORES, 1987). El niño prefiere que sus resultados sean evaluados por los adultos, lo cual es consecuencia de que el escolar no domina los parámetros a través de los cuales puede autoevaluarse, situación que se transforma progresivamente cuando aprende cuáles son los indicadores necesarios para valorar su conducta y sus resultados. También en nuestro país P. Rico (1987) demostró que el desarrollo del autocontrol, a partir de la utilización por parte de los niños de criterios objetivos para valorar su trabajo, favorece el desarrollo de la autovaloración y la calidad de la ejecución de las tareas. A su vez, A. Amador (1987), constató la influencia que ejercen sobre el desarrollo de la autovaloración las interrelaciones del alumno en su grupo escolar y la estrecha relación de su adecuación con la posición favorable o desfavorable ocupada por el niño en el grupo. Sobre este último aspecto, F. González (1982b) destaca como la valoración social constituye una importante vía de formación de la autovaloración. Esta 
valoración cuando se centra en los resultados obtenidos por el escolar, al margen de sus cualidades personales: es decir, del esfuerzo empleado y de las acciones emprendidas para el logro de determinados objetivos, puede conducir al surgimiento de una autovaloración inadecuada, ya sea por sobrevaloración o por sub-valoración que repercutirá desfavorablemente en el aprendizaje y en toda la vida de relación del sujeto con quienes le rodean.

En la adolescencia una cuestión central para el adolescente es lograr responderse la pregunta de ¿quién soy?, por lo que la autovaloración puede considerarse como la neoformación correspondiente a esta etapa. En el desarrollo de la autovaloración influyen diferentes condiciones como son las transformaciones puberales y su repercusión psicológica, especialmente en la imagen de sí, y en particular, en la imagen corporal; las nuevas exigencias propias de los sistemas de actividad y comunicación típicos de estos períodos y el desarrollo intelectual, asociado a las necesidades de independencia, autoafirmación y comunicación, aún cuando a veces el adolescente tiende a la soledad y al aislamiento para reflexionar sobre sí mismo. En la Adolescencia la imagen corporal y las cualidades vinculadas a las relaciones interpersonales tienen un marcado peso en la autovaloración y en el sentido de autoestima. En este período se integran las funciones subjetivo-valorativa y reguladora de la autovaloración (ROLOFF, 1987). En la Adolescencia se alcanza un nivel cualitativamente superior de desarrollo de la autoconciencia (BOZHOVICH, 1976), la cual adquiere un carácter generalizado y sirve de sostén al desarrollo de la autovaloración. No obstante, esta aún es inestable e inexacta, pues depende en determinada medida de las valoraciones externas. En sus relaciones interpersonales el adolescente tiende a veces a sobrevalorarse o a considerar que los demás no tienen una valoración adecuada de su persona, ya sea por desconocimiento o porque subvaloran sus cualidades, cuestión que según Kon (1990) a veces es cierta en el caso de los maestros.

En la juventud la autovaloración resulta más flexible y estructurada que en la adolescencia, lo que indica que esta formación ha ganado en estabilidad y objetividad, sirviendo de apoyo a la elaboración del sentido de la vida o proyecto de vida. El joven confiere mayor importancia a aquellas cualidades vinculadas con el dominio de sí mismo y su proyección futura. La función reguladora de la autovaloración se transforma en autoeducativa, orientando el comportamiento del joven, en su proyección presente y futura, hacia su auto 
perfeccionamiento. Esta función autoeducativa le brinda al joven la posibilidad de proponerse tareas dirigidas a su propio perfeccionamiento y de distintas estrategias orientadas al logro de este propósito.

\subsubsection{El desarrollo moral}

En cuanto al desarrollo moral, en la edad escolar se crean las condiciones para que el niño adquiera determinadas cualidades morales que se convierten en motivos estables de su conducta. Apunta Bozhovich (1976) que para que se produzca este proceso deben estar presentes determinadas condiciones. En primer lugar, que el niño posea un motivo suficientemente fuerte y estable que lo estimule o impulse a poner en práctica determinado comportamiento acorde a la cualidad. En segundo lugar, es imprescindible enseñar al niño aquellas formas de conducta que se corresponden con la cualidad en cuestión, ya que no basta con que el niño se sienta motivado a comportarse de determinada forma, sino que necesita dominar determinados hábitos que le permitan expresar dicha cualidad en su comportamiento. Para lograrlo, la autora recomienda que se le brinden al escolar los medios externos que faciliten la formación e interiorización de la cualidad deseada, en los cuales inicialmente pueda apoyarse.

En la adolescencia una de las principales características del desarrollo moral es que las cuestiones relacionadas con las reglas y normas vinculadas a las relaciones interpersonales y a la conducta social en general, a criterio de L. I. Bozhovich (1976), ocupan un lugar importante en las reflexiones del adolescente. De acuerdo con lo antes señalado, observamos que el adolescente posee puntos de vista, juicios y opiniones de carácter moral que presentan una relativa estabilidad e independencia de las influencias externas, pero al mismo tiempo estas valoraciones morales no se han convertido aún en convicciones, por lo que pueden ser modificadas en determinados contextos, en especial, bajo la opinión de los coetáneos. Esta regulación moral, determinada todavía en última instancia por exigencias externas, fue denominada por J. Piaget (1971) como moral heterónoma y por su seguidor L. Kohlberg (1978) como moral convencional.

En la juventud como expresión del desarrollo moral vemos que los conceptos morales se hacen más conscientes y estructurados y el joven logra formularlos correctamente a través del lenguaje. En esta etapa la moral 
opera desde lo interno. El sujeto asume la responsabilidad personal de sus acciones basándose en principios morales generales y, a la vez, logra una mayor flexibilidad y argumentación de sus valoraciones morales. Así, la tendencia a la autoafirmación del adolescente, se sustituye, en opinión de Kon (1990), por un auto-análisis más realista y crítico y por la autoeducación de la personalidad, que desde la concepción de Piaget (1971) se traduce en una moral autónoma y de principios auto-aceptados para Kohlberg (1978).

La regulación moral en la Juventud resulta más estable, en comparación con la alcanzada etapa de la adolescencia, y esto se debe, en buena medida, al surgimiento de la concepción del mundo, considerada por los autores marxistas como neoformación que distingue a esta edad. La concepción del mundo es la representación generalizada y sistematizada de la realidad en su conjunto, de las leyes que rigen su devenir y de las exigencias que plantea el medio social a la actuación del joven; es también la representación del lugar que ocupa el hombre en este contexto, y por ende, la propia personalidad.

La concepción del mundo sirve de sostén a la elaboración del sentido de la vida, en tanto sentido de la propia existencia y estrategia a seguir para encontrar el lugar al que se aspira en el cuadro del mundo.

\subsubsection{Ideales y proyectos futuros}

Los ideales, formación que también tributa a la regulación moral en la edad escolar, son definidos como modelos de gran significación emocional para el niño, ya que se convierten en patrones de evaluación de su propia conducta y de otras personas. En esta etapa los ideales presentan un carácter concreto. El modelo elegido se trata generalmente de alguna persona cercana al niño, fundamentalmente alguno de sus padres. Este modelo se acepta en su totalidad, sin llevarse a cabo una evaluación crítica del mismo, siendo inseparable de la situación en la que actúa. Su elección por parte del escolar es más emocional que racional.

Los ideales definidos por Bozhovich (1976) como modelos emocionalmente matizados e interiormente aceptados que se convierte en criterio de valoración de la conducta propia y de la de otros, se encuentran representados la adolescencia, según la autora, por personajes de la Literatura o de la Historia que han mostrado su valor y autodominio. Es por ello que comienzan a tener un carácter abstracto, en comparación con la Edad Escolar, etapa 
en la que se caracterizaban por ser concretos. Sin embargo, por existir una tendencia propia de la edad a destacar en el ideal cualidades de carácter moral muy elevadas, a veces los adolescentes no logran o les resulta difícil organizar su conducta atendiendo a las cualidades seleccionadas.

En la juventud se produce una búsqueda consciente del ideal, el cual se asume teniendo como base una valoración moral y crítica de sus características. Los ideales, al igual que la autovaloración, se convierten en patrón de evaluación del comportamiento del joven propio y ajeno. De esta forma, encontramos como ideales típicos de este período los llamados ideales generalizados, ya que generalmente el joven no elige un modelo concreto o representado por una persona particular, sino que el contenido del ideal coincide con su proyección futura aunque pueden ser formales o efectivos (GONZÁLEZ, 1982 b). Incluso, puede prevalecer un ideal cuya estructura está asociada a un modelo concreto, pero el mismo se asume críticamente y con un alto nivel de elaboración, ya que se abstraen y fundamentan aquellas cualidades del modelo que responden al sistema de necesidades, motivos y exigencias morales, asumidas por el joven. Este tipo de ideal fue denominado por Grichanova (GONZÁLEZ, 1982b) como "ideal concretizado".

En la Juventud se produce una estrecha interrelación entre la identidad personal, los valores y el proyecto de vida (DOMÍNGUEZ, 2003b). En un artículo, escrito en colaboración con L. Ibarra, titulado Juventud y proyectos de vida (DOMÍNGUEZ, 2003a, p. 446), entendemos por proyección futura "la estructuración e integración de un conjunto de motivos, elaborados en una perspectiva temporal futura, a mediano o largo plazo, que poseen una elevada significación emocional o sentido personal para el sujeto y de las estrategias correspondientes para el logro de los objetivos propuestos".

Desde 1998 hasta la fecha, en trabajos investigativos de diploma y maestría realizados bajo mi tutoría en torno al desarrollo de los proyectos futuros en jóvenes cubanos (DOMÍNGUEZ, 2014) se obtuvieron interesantes resultados sobre el tema. En estos trabajos hemos partido de cuatro categorías de análisis que fueron siendo construidas en el propio proceso investigativo y cada una de las cuales es evaluada por distintos indicadores. Dichas categorías son: contenido del proyecto: Esfera de los sistemas de actividad o de comunicación al que se orienta. También puede estar referido a la propia persona, estrategias: Vías o acciones a partir de las cuales el sujeto piensa alcanzar los proyectos a los que aspira. Pueden presentarse 
como estructuradas, parcialmente estructuradas o no estructuradas, temporalidad: Momento en el tiempo en que se ubica la consecución del proyecto; es decir, si a corto plazo (hasta un año), mediano plazo (más de un año y hasta cinco años), largo plazo (más de cinco años) o plazo indefinido y obstáculos: previsión de contingencias que puedan afectar la consecución de los proyectos; ya sean de carácter interno (dependen de la intencionalidad del sujeto), externo (son independientes de la voluntad del sujeto) o mixtos (cuando se mencionan a la vez obstáculos internos y externos).

\subsubsection{Intereses cognoscitivos e intereses profesionales}

En los primeros grados los intereses cognoscitivos están muy relacionados con la aprobación del maestro y tienden a orientarse hacia el proceso de estudio. Amediados de la edad escolar el niño comienza a interesarse por los resultados de sus acciones y, en la medida en que se hace consciente de sus progresos y de la utilidad de los conocimientos, continúan desarrollándose sus intereses. Ya en los grados terminales los intereses no se dirigen únicamente al conocimiento de determinados hechos, sus causas y las relaciones entre ellos, sino también hacia la solución de problemas, aspecto este último que se consolida en la etapa de la adolescencia, mientras en la Juventud estos intereses estimulan al sujeto al planteamiento de nuevos problemas.

En el transcurso de la adolescencia el problema de la elección de la futura profesión aún no ocupa un lugar central en las reflexiones del adolescente, y aunque pueden existir algunos intereses profesionales surgidos sobre la base de los intereses cognoscitivos y docentes, no necesariamente constituyen expresión de una motivación profesional estructurada. En sentido general, la elección de la profesión no se realiza como un verdadero acto de autodeterminación, que implique una decisión consciente e intencional del sujeto. Esta elección en la adolescencia se asocia en buena medida al prestigio social de las profesiones o al vínculo de estas con determinadas asignaturas preferidas.

En la juventud se desarrollan intereses profesionales y la elección profesional se realiza basada en la motivación profesional del sujeto -término empleado en nuestra tesis de doctorado (1992), basada en las consideraciones de F. González (1983a). Dicha elección debe acontecer como acto de autodeterminación ya que el joven está en condiciones de realizar una valoración de sus intereses, capacidades y posibilidades, partiendo de sus 
aspiraciones, y en correspondencia con esta evaluación tomar una decisión más fundamentada, aunque esta elección puede efectuarse también por mecanismos psicológicos totalmente diferentes, como son la búsqueda de prestigio social, de aprobación familiar, de bienestar económico, la necesidad de ser útil a la sociedad, etc. (DOMÍNGUEZ, 2003c).

\section{Consideraciones finales}

En la edad escolar, la adolescencia y la juventud las variaciones que se producen en el contenido y los métodos de obtención del conocimiento en el estudio -como actividad formal o institucionalizada-influirán notablemente en el desarrollo de la personalidad.

En cuanto a la actividad informal o no institucionalizada constatamos que se desarrollan diversas actividades en el tiempo libre, las cuales se seleccionan de manera más intencional en la adolescencia -aunque muy vinculadas a los intereses del grupo de coetáneos- y en la juventud como elección personalizada, en comparación con la edad escolar.

El sistema de comunicación en las edades en cuestión presenta determinadas características, las cuales permiten captar el movimiento de continuidad y ruptura propio de este proceso:

La comunicación con los adultos durante la edad escolar se basa en que son considerados por el niño como figuras sagradas, mientras que en la Adolescencia se produce una relación polémica, donde el carácter dicotómico del pensamiento del adolescente y todas las características de su "situación social del desarrollo" favorecen los conflictos con los adultos, quienes por desconocimiento de las particularidades de la edad y de las condiciones que la provocan mantienen actitudes de intolerancia desde posiciones de fuerza que avivan este conflicto y las manifestaciones externas de la crisis. En la Juventud, gracias a la madurez alcanzada por el joven, al surgimiento de su concepción del mundo y a la estructuración del sentido de la vida, la comunicación con los adultos se basa en el respeto mutuo, y la aceptación pasa por una actitud crítica pero a la vez reflexiva del joven, lo que favorece el intercambio comunicativo sobre nuevas bases.

La comunicación con los coetáneos o iguales se establece a partir de la inserción de escolares, adolescentes y jóvenes en grupos organizados e informales. En la edad escolar las normas y valores que regulan las relacio- 
nes entre los miembros del grupo de iguales poseen un carácter externo. En la etapa de adolescencia, se deriva de la opinión social imperante entre los miembros del grupo, y sólo llegan a operar desde lo interno como proceso autorregulado en la etapa de la juventud, gracias al surgimiento de la concepción del mundo como neoformación que tipifica a ese período.

Las relaciones de amistad poco profundas, inestables y casuales en la edad escolar, pasan en la adolescencia por una etapa de idealización y romanticismo, carente de una visión crítica que oriente estos intercambios. En la Juventud se crean las premisas para que las relaciones de amistad ganen en profundidad, estabilidad y madurez, dada la capacidad de autodeterminación del joven y la aparición de la concepción del mundo, con su fuerte contenido moral.

Las relaciones de pareja, prácticamente inexistentes en la edad escolar, cobran gran importancia en la adolescencia cono medio de autoconocimiento y autoafirmación, aunque tienden a ser promiscuas e inestables. En la juventud estas relaciones ganan en estabilidad y se comienza a poner en práctica el proyecto de constitución de la propia familia.

Las regularidades aquí analizadas basándonos en la categoría "situación social del desarrollo" se presentan como tendencias y permiten establecer los límites de estas edades, sin desconocer que las mismas se expresan en el sujeto individual de manera particular e irrepetible, en tanto dependen de las condiciones de educación en las que transcurre su vida, de las principales adquisiciones psicológicas logradas en las etapas anteriores y, muy especialmente, de la forma en que él se apropia de las influencia externas y construye activamente su propia subjetividad.

El análisis de estas regularidades debe aún profundizarse desde una visión dialéctica de la relación existente entre los diferentes factores determinantes del desarrollo psicológico, que nos permita una comprensión más amplia y profunda del carácter histórico y social de los diferentes períodos del ciclo vital de desarrollo humano, desde una perspectiva psicológica. Esta tarea constituye una premisa esencial para lograr hacer realidad en la práctica pedagógica y en el proceso de educación de la personalidad, el principio vygotskiano de que la enseñanza precede el desarrollo y lo dirige. 


\section{Referencias}

ÁGUILA, R. Y. Proyectos profesionales y utilización del tiempo. 2005. 118f. Tesis de Diploma (Graduación en Psicología) - Facultad de Psicología. Universidad de La Habana, Cuba. 2005.

BOZHOVICH, L. I. Las etapas de formación de la personalidad en la ontogénesis. In: . La psicología evolutiva y pedagógica en la URSS. Antología. Moscú: Editorial Progreso. 1987.

CARRETERO, M.; LEÓN, J. A. Del pensamiento formal al cambio conceptual en la adolescencia”. In: PALACIOS, J.; MARCHESI, Á.; COLL, C. (Comp.) Desarrollo psicológico y educación. Cap. 19, p. 453-469. Madrid: Alianza Editorial S.A. 1999.

COLECTIVO DE AUTORES. Psicología del desarrollo del escolar. Selección de Lecturas. Tomo I. Compiladoras: Ana Luisa Segarte Iznaga, Graciela A. Martínez Campos y María Emilia Rodríguez Pérez. La Habana: Editorial "Félix Varela", 2003.

. Investigaciones acerca de la formación de las nuevas generaciones. La Habana: Editora Universitaria. 1990.

. Investigaciones de psicología pedagógica acerca del escolar cubano. La Habana: Editorial Pueblo y Educación, 1987.

DAVIDOV, V. V. Tipos de generalización en la enseñanza. Editorial Pueblo y Educación: Cuba. 1981.

DOMÍNGUEZ, G. L. Proyectos futuros en jóvenes cubanos. Trabajo presentado en el Evento Internacional Universidad 2014. La Habana, Cuba, 2014.

. Psicología del desarrollo. Problemas, principios y categorías. La Habana: Editorial "Félix Varela", 2007.

. Psicología del desarrollo: adolescencia y juventud. Selección de lecturas. Compiladora: Laura Domínguez García. La Habana: Editorial "Félix Varela", 2003a.

. Identidad, valores y proyecto de vida. In: RIUS, L. F. (Comp.) Pensando en la personalidad. Tomo II. p. 93-99. La Habana: Editorial Félix Varela. 2003b. 
. Motivación profesional y personalidad. In: . Pensando en la personalidad. Tomo I. p. 366-415. La Habana: Editorial Félix Varela, 2003c. . Caracterización de los niveles de desarrollo de la motivación profesional en jóvenes estudiantes. 1992. 245f. Tesis (Doctorado en Ciencias Pedagógicas) - Facultad de Psicología, Universidad de La Habana, Cuba, 1992.

ELKONIN, D. B. La psicología evolutiva y pedagógica en la URSS. Antología Moscú: Editorial Progreso, 1987.

GALPERIN, P. Y. Sobre la investigación del desarrollo intelectual del niño. In: . La psicología evolutiva y pedagógica en la URSS. Antología. Moscú: Editorial Progreso, 1987.

GONZÁLEZ, R. F. Motivación profesional en adolescentes y jóvenes. La Habana: Editorial Ciencias Sociales, 1983a.

. Motivación moral en adolescentes y jóvenes. La Habana: Editorial Científico -Técnica, 1983b.

HERNÁNDEZ, D. Y. Proyectos futuros y empleo del tiempo en estudiantes universitarios. 2006. 173f. Tesis de Diploma (Graduación en Psicología) Facultad de Psicología, Universidad de La Habana, Cuba, 2006.

HUMARAN, B. Y. Caracterización de la repercusión psicológica de las transformaciones puberales en adolescentes que residen en distintos contextos socioeconómicos. 2009. 165f. Tesis de Diploma. (Graduación en Psicología) - Facultad de Psicología, Universidad de La Habana, Cuba, 2009.

KOHLBERG, L. Revisiones en la teoría y práctica del desarrollo moral. San Francisco: Editorial Jossey-Bass, 1978.

KON, I. S. Psicología de la edad juvenil. La Habana: Editorial Pueblo y Educación, 1990.

PETROVSKY, A. V. Psicología evolutiva y pedagógica. Moscú: Editorial Progreso, 1980.

PIAGET, J. El criterio moral en el niño. Barcelona: Editorial Fontanella, 1971. 
. El nacimiento de la inteligencia en el niño. Barcelona: Editorial Aguilar S.A. 1969.

.; WALLON, H. Los estadios en la psicología del niño. La Habana: Edición Revolucionaria, 1966.

RUBINSTEIN, S. L. Principios de psicología general. México: Editorial Grijalbo, S. A., 1967.

VYGOTSKI, L. S. El desarrollo de las funciones psíquicas superiores. La Habana: Editorial Científico Técnica. 1987.

. El problema de la edad. In: . Problemas de la psicología infantil. Cap. V. Moscú: Editorial Pedagógica, 1984.

. Pensamiento y lenguaje. La Habana: Editorial Pueblo y Educación. 1982.

. El problema del entorno. In: Fundamentos de la podología. Cuarta Conferencia publicada. Leningrado: Izdanie Instituto, 1935.

YEMI, G. G. Caracterización de las repercusiones psicológicas referidas a los cambios fisicos en un grupo de adolescentes cubanos. 2006. $120 \mathrm{f}$. Tesis de Diploma. (Graduación en Psicología) - Facultad de Psicología, Universidad de La Habana, 2006.

Data de registro: $21 / 10 / 2014$

Data de aceite: 22/04/2015 